\title{
ON THE TRICYCLIC GRAPHS WITH THREE DISJOINT 6-CYCLES AND MAXIMUM MATCHING ENERGY
}

\author{
YUN-XIA ZHOU AND HONG-HAI LI
}

\begin{abstract}
The matching energy of a graph was introduced recently by Gutman and Wagner and defined as the sum of the absolute values of zeros of its matching polynomial. In this paper, we characterize graphs that attain the maximum matching energy among all connected tricyclic graphs of order $n$ with three vertex-disjoint $C_{6}$ 's.
\end{abstract}

\section{Introduction}

A matching in a graph is a set of pairwise nonadjacent edges, and by $m_{k}(G)$ we denote the number of $k$-matchings of a graph $G$. It is both consistent and convenient to define $m_{0}(G)=1$. In 2012, Gutman and Wagner [5] introduced the matching energy of a graph $G$, denoted by $M E(G)$, as

$$
M E(G)=\frac{2}{\pi} \int_{0}^{\infty} \frac{1}{x^{2}} \ln \left[\sum_{k \geq 0} m_{k}(G) x^{2 k}\right] d x,
$$

which extends the formula for energy for forests to general graphs (for more on graph energy see the monograph [12] and [6, 7, 4] and the references therein). The integral on the right hand side of Eq. (1) is increasing in all of the coefficients $m_{k}(G)$. This means that if two graphs $G$ and $G^{\prime}$ satisfy $m_{k}(G) \leq m_{k}\left(G^{\prime}\right)$ for all $k \geq 1$, then $M E(G) \leq M E\left(G^{\prime}\right)$. If, in addition, $m_{k}(G)<m_{k}\left(G^{\prime}\right)$ for at least one $k$, then $M E(G)<M E\left(G^{\prime}\right)$. It then motivates the introduction of a quasi-order $\geq$, defined by

$$
G \geq H(\text { or } H \leq G) \Longleftrightarrow m_{k}(G) \geq m_{k}(H), \quad \text { for all nonnegative integers } k \text {. }
$$

If $G \geq H$ and there exists some $k$ such that $m_{k}(G)>m_{k}(H)$, then we write $G>H$ (or $H \prec G$ ). We have $G \geq H \Longrightarrow M E(G) \geq M E(H)$ and $G>H \Longrightarrow M E(G)>M E(H)$. From the definition it Received October 31, 2014, accepted April 24, 2015. 2010 Mathematics Subject Classification. 05C50.

Key words and phrases. Tricyclic graph, matching energy, k-matching, matching polynomial. Corresponding author: Hong-Hai Li.

Supported by National Natural Science Foundation of China (11561032, 11201198), Natural Science Foundation of Jiangxi Province (20142BAB211013), the Sponsored Program for Cultivating Youths of Outstanding Ability in Jiangxi Normal University. 
is clear that if $H$ is a subgraph of $G$ such that the edge set of $H$ is a proper subset of the edge set of $G$, then $G>H$.

Recall that the matching polynomial of a graph $G$ of order $n$ is defined as

$$
\alpha(G, x)=\sum_{k \geq 0}(-1)^{k} m_{k}(G) x^{n-2 k},
$$

where the convention that $m_{k}(G)=0$ for $k<0$ or $k>n / 2$ is adopted. For any graph $G$, all the zeros of $\alpha(G, x)$ are real-valued.

Theorem 1.1 ([5]). Let $G$ be a simple graph, and let $\mu_{1}, \mu_{2}, \ldots, \mu_{n}$ be the zeros of its matching polynomial. Then

$$
\operatorname{ME}(G)=\sum_{i=1}^{n}\left|\mu_{i}\right| .
$$

Given a graph $G$ and an edge $u v$ of $G$, we denote by $G-u v$ (resp. $G-v$ ) the graph obtained from $G$ by deleting the edge $u v$ (resp. the vertex $v$ and the edges incident to it).

Lemma 1.2 ([3]). If $u, v$ are adjacent vertices of $G$, then for all nonnegative integers $k$, we have

$$
m_{k}(G)=m_{k}(G-u v)+m_{k-1}(G-u-v)
$$

Denote by $\mathscr{U}_{g, n}$ the set of unicyclic graphs with $n$ vertices and a cycle of length $g$. The sun graph, denoted by $C_{g}\left(P_{r_{1}+1}, \ldots, P_{r_{g}+1}\right)$, is the graph obtained from the cycle $C_{g}=v_{1} v_{2} \cdots v_{g} v_{1}$ by identifying one pendant vertex of the path $P_{r_{i}+1}$ with vertex $v_{i}$ for $i=1, \ldots, g$. Note that $C_{g}\left(P_{n-g+1}, P_{1}, \ldots, P_{1}\right)$ is also called lollipop graph and is denoted by $E_{g, n}$. For convenience, we adopt the convention that $E_{g, n}=C_{n}$ when $g=n$.

The proof for Lemma 3.8 in [9] actually establishes the following slightly stronger result.

Lemma 1.3. Let $n, g$ be any positive integers, $n>g \geq 3$. For any $G \in \mathscr{U}_{g, n} \backslash\left\{E_{g, n}\right\}$, we have $E_{g, n}>G$.

We provide a simple, different proof for the following result which slightly strengthens Lemma 5 of [3].

Lemma 1.4 ([3]). Let $u, v$ be adjacent vertices of a graph $G$. If $G_{1}\left(\right.$ resp., $\left.G_{2}\right)$ is the graph obtained from $G$ by inserting $t$ vertices into the edge $u v$ (resp., by joining the vertex $u$ to an end vertex of a path $P_{t}$ ) then $G_{1} \geq G_{2}$. If in addition $d_{G}(u) \geq 2$, then $G_{1}>G_{2}$.

Proof. Let $v_{p}$ (resp., $v_{q}$ ) denote the vertex on the path in $G_{1}$ joining $u$ and $v$, adjacent to $u$ (resp., $v$ ). It is easy to see that the graphs $G_{1}-v_{q} v$ and $G_{2}-u v$ are isomorphic. Moreover, $G_{2}-u-v$ is isomorphic with a graph, say $H$, obtained from $G_{1}-v_{q}-v$ by deleting all edges in 
$G_{1}$ incident with $u$, except for the edge $u v_{p}$. If $d_{G}(u) \geq 2$, the edge set of $H$ is proper subset of that of $G_{1}-v_{q}-v$. By Lemma 1.2, for any positive integer $k$, we have $m_{k}\left(G_{2}\right)=m_{k}\left(G_{2}-u v\right)+$ $m_{k-1}\left(G_{2}-u-v\right)=m_{k}\left(G_{1}-v_{q} v\right)+m_{k-1}(H) \leq m_{k}\left(G_{1}-v_{q} v\right)+m_{k-1}\left(G_{1}-v_{q}-v\right)=m_{k}\left(G_{1}\right)$, where the inequality strictly holds for at least one $k$ if $d_{G}(u) \geq 2$, because then the edge set of $H$ is a proper subset of that of $G_{1}-v_{q}-v$. Hence our result follows.

We adopt the convention that $P_{0}$ stands for the null graph. So $P_{0} \cup G=G$ for any graph $G$.

Lemma 1.5 ([12]). Let $n$ be a given positive integer, and let $l, l^{\prime}$ be nonnegative integers less than or equal to $\lfloor n / 2\rfloor$. We have

(i) If $l, l^{\prime}$ are even and $l<l^{\prime}$ then $P_{l} \cup P_{n-l}>P_{l^{\prime}} \cup P_{n-l^{\prime}}$.

(ii) If $l, l^{\prime}$ are odd and $l<l^{\prime}$ then $P_{l} \cup P_{n-l}<P_{l^{\prime}} \cup P_{n-l^{\prime}}$.

(iii) If $l$ is even and $l^{\prime}$ is odd, then $P_{l} \cup P_{n-l}>P_{l^{\prime}} \cup P_{n-l^{\prime}}$.

In[5], Gutman and Wagner first introduced the notion of matching energy and characterized the extremal (maximal or minimal with respect to matching energy) graphs among some special graph classes. Li and Yan [11] characterized the connected graph with the given connectivity (resp. chromatic number) which has maximum matching energy. Ji, Li and Shi [8] characterized the graphs with the extremal matching energy among all bicyclic graphs. Recently, Chen et.al [1, 2] further investigated unicyclic graphs, bicyclic graphs and tricyclic graphs for extremal matching energy. For more on matching energy see[10, 14] and the references therein.

In [13], Li, Shi and Wei characterized graphs that attain the maximal energy among all connected tricyclic graphs on $n$ vertices with three disjoint cycles. In this paper, we treat a similar problem for the matching energy, i.e, we characterize graphs that attain the maximum matching energy among all connected tricyclic graphs of order $n$ with three vertex-disjoint cycles of length 6 .

\section{Main results}

We borrow part of the definitions and notations from [13]. We say $H$ is the central structure of $G$ if $G$ can be obtained from $H$ by planting some trees on it. Let $G_{6, n}$ denote the set of all connected tricyclic graphs on $n$ vertices with three disjoint $C_{6}$ 's. Given positive integers $l_{1}, l_{2}, l_{3}$ such that $l_{1}, l_{2} \geq 2$ and $1 \leq l_{3} \leq 4$, let $\Phi_{6}^{I}\left(l_{1}, l_{2} ; l_{3}\right)$ denote the graph in $G_{6, n}$, as shown in Figure 1 , where the first $C_{6}$ and the second $C_{6}$ are joined by a path $P_{1}=v \cdots u$ on $l_{1}$ vertices, the second $C_{6}$ and the third $C_{6}$ are joined by a path $P_{2}=u^{\prime} \cdots w$ on $l_{2}$ vertices, the smaller part $u \cdots u^{\prime}$ of the second $C_{6}$ has $l_{3}$ vertices. Note that when $u=u^{\prime}$, we have $l_{3}=1$. Similarly, given positive integers $l_{1}, l_{2}, l_{3} \geq 2$ and $n=l_{1}+l_{2}+l_{3}+13$, let $\Phi_{6}^{\Pi}\left(l_{1}, l_{2}, l_{3}\right)$ denote the 
graph as shown in Figure 1: it has a center vertex $v$ such that the three cycles $C_{6}$ are joined to $v$ by paths $P_{1}=u_{1} \cdots v, P_{2}=u_{2} \cdots v, P_{3}=u_{3} \cdots v$ with $l_{1}, l_{2}$ and $l_{3}$ vertices, respectively. Note that $\Phi_{6}^{I}\left(l_{1}, l_{2} ; l_{3}\right)=\Phi_{6}^{I}\left(l_{2}, l_{1} ; l_{3}\right)$ and $\Phi_{6}^{\Pi}\left(l_{1}, l_{2}, l_{3}\right)=\Phi_{6}^{\Pi}\left(l_{\sigma(1)}, l_{\sigma(2)}, l_{\sigma(3)}\right)$ for any permutation $\sigma \in S_{3}$. Denote by $\Phi_{6, n}^{I}$ (resp., $\Phi_{6, n}^{\Pi}$ ) the set of all graphs of the form $\Phi_{6}^{I}\left(l_{1}, l_{2} ; l_{3}\right)$ (resp., $\left.\Phi_{6}^{\Pi}\left(l_{1}, l_{2}, l_{3}\right)\right)$ for some $l_{1}, l_{2}, l_{3}$. Denote by $G_{6, n}^{I}\left(\right.$ resp., $\left.G_{6, n}^{\Pi}\right)$ the set of all graphs in $G_{6, n}$ that
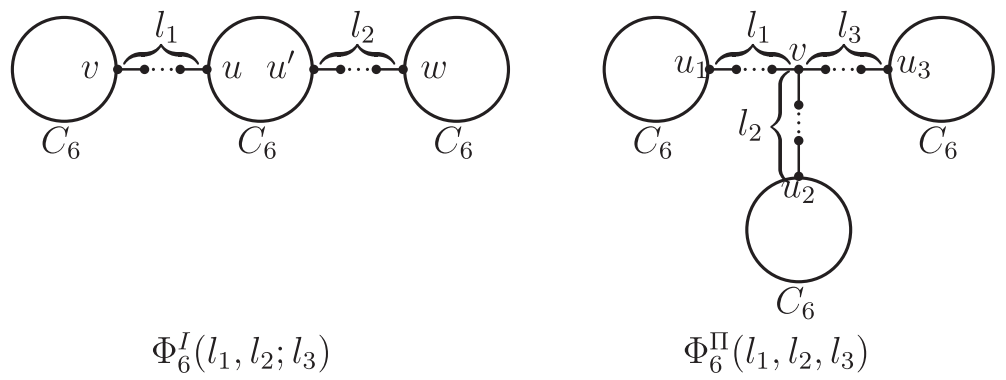

$$
\Phi_{6}^{I}\left(l_{1}, l_{2} ; l_{3}\right)
$$$$
\Phi_{6}^{\Pi}\left(l_{1}, l_{2}, l_{3}\right)
$$

Figure 1: $\Phi_{6}^{I}\left(l_{1}, l_{2} ; l_{3}\right)$ and $\Phi_{6}^{\Pi}\left(l_{1}, l_{2}, l_{3}\right)$.

have $\Phi_{6}^{I}\left(l_{1}, l_{2} ; l_{3}\right)\left(\right.$ resp., $\left.\Phi_{6}^{\Pi}\left(l_{1}, l_{2}, l_{3}\right)\right)$ for some appropriate $l_{1}, l_{2}, l_{3}$ as their central structures. Now note that $G_{6, n}=G_{6, n}^{I} \cup G_{6, n}^{\Pi}$.

Lemma 2.1. Let $u v$ be a bridge of a connected graph $G$ such that $G_{1}, H$ are the connected components of $G-u v$ with $u \in V\left(G_{1}\right)$ and $v \in V(H)$. Let $G_{2}$ be a graph, vertex-disjoint from $H$, that satisfies $G_{2} \geq G_{1}$. Let $G^{\prime}$ be the graph obtained from $G_{2}, H$ by adding an edge joining $v$ to some vertex $u^{\prime}$ of $G_{2}$. Suppose that $G_{2}-u^{\prime} \geq G_{1}-u$. Then $G^{\prime} \geq G$. If, in addition, one of the quasi-inequalities $G_{2} \geq G_{1}$ and $G_{2}-u^{\prime} \geq G_{1}-u$ is strict, then $G^{\prime}>G$.

Proof. By Lemma 1.2, for every positive integer $k$, we have $m_{k}(G)=m_{k}(G-u v)+m_{k-1}(G-$ $u-v)=m_{k}\left(G_{1} \cup H\right)+m_{k-1}\left(\left(G_{1}-u\right) \cup(H-v)\right)$ and $\left.m_{k}\left(G^{\prime}\right)=m_{k}\left(G_{2} \cup H\right)+m_{(} k-1\right)\left(\left(G_{2}-\right.\right.$ $\left.\left.u^{\prime}\right) \cup(H-v)\right)$. Since $G_{2} \geq G_{1}$ and $G_{2}-u^{\prime} \geq G_{1}-u$, we have $m_{k}\left(G_{2} \cup H\right) \geq m_{k}\left(G_{1} \cup H\right)$ and $m_{k-1}\left(\left(G_{2}-u^{\prime}\right) \cup(H-v)\right) \geq m_{k-1}\left(\left(G_{1}-u\right) \cup(H-v)\right)$; hence $m_{k}\left(G^{\prime}\right) \geq m_{k}(G)$. This establishes the quasi-inequality $G^{\prime} \geq G$. It is clear that the latter quasi-inequality is strict if one of the quasi-inequalities $G_{2} \geq G_{1}$ and $G_{2}-u^{\prime} \geq G_{1}-u$ is strict.

Lemma 2.2. For any $G \in G_{6, n}^{I} \backslash \Phi_{6, n}^{I}, G \prec \Phi_{6}^{I}\left(l_{1}, l_{2} ; 2\right)$ for some positive integers $l_{1}, l_{2} \geq 2$ that satisfy $l_{1}+l_{2}=n-14$.

Proof. By Lemma 1.4 we may assume that $G$ has no nontrivial rooted tree, where the root is an internal vertex of one of the two paths joining the $C_{6}$ 's. So we may assume that $G$ is of the form as shown in Figure 2, which can be obtained by connecting sun graphs $C_{6}\left(P_{r_{1}+1}, \ldots, P_{r_{5}+1}, P_{1}\right)$ and $\mathscr{S}$ by a path $P_{l_{1}}, \mathscr{S}$ and $C_{6}\left(P_{1}, P_{t_{1}+1}, P_{t_{2}+1}, \ldots, P_{t_{5}+1}\right)$ by a path $P_{l_{2}}$, where the middle sun 


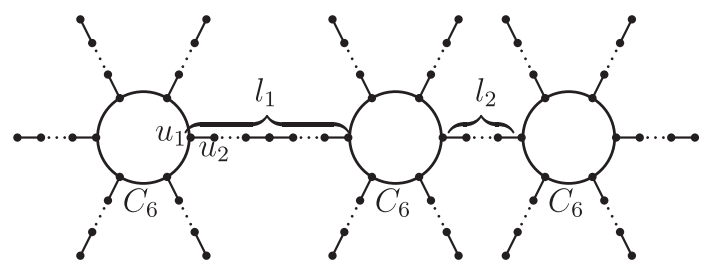

Figure 2: $G$ when $l_{3}=3$.

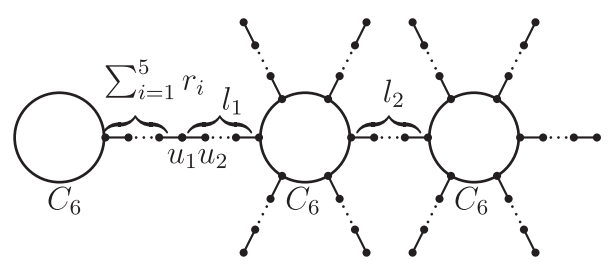

Figure 3: $G^{\prime}$.

graph $\mathscr{S}$ has either five pendent paths of length $s_{1}, s_{2}, s_{3}, s_{4}, s_{5}$ or four pendent paths of length $s_{1}, s_{2}, s_{3}, s_{4}$ according to $l_{3}=1$ or not.

If $\sum_{i=1}^{5} r_{i} \geq 1$, then by applying Lemma 2.1 with $G_{1}$ equal to $C_{6}\left(P_{r_{1}+1}, \ldots, P_{r_{5}+1}, P_{1}\right), H$ equal to the graph obtained from $G$ by deleting $V\left(G_{1}\right)$ and all incident edges, $G^{\prime}$ equal to the graph as shown in Figure 3, $G_{2}$ equal to $E_{6, r_{1}+r_{2}+\cdots+r_{5}+6}$ and $u=u^{\prime}=u_{1}, v=u_{2}$, and noting that $C_{6}\left(P_{r_{1}+1}, \ldots, P_{r_{5}+1}, P_{1}\right) \prec E_{6, r_{1}+r_{2}+\cdots+r_{5}+6}$ by Lemma 1.3 and $C_{6}\left(P_{r_{1}+1}, \ldots, P_{r_{5}+1}, P_{1}\right)-u_{1} \prec$ $P_{r_{1}+r_{2}+\cdots+r_{5}+5} \prec E_{6, r_{1}+r_{2}+\cdots+r_{5}+5}$, we obtain $G \prec G^{\prime}$.

Similarly, if $\sum_{i=1}^{5} t_{i} \geq 1$, then by applying Lemma 2.1, we can also show that $G \prec G^{\prime}$ for some $G^{\prime} \in \Phi_{6, n}^{I}$. So we can assume that $G$ is obtained from some $\Phi_{6}^{I}\left(l_{1}^{\prime}, l_{2}^{\prime} ; l_{3}\right)$ by attaching a pendant path at each vertex of degree two in the middle cycle; say, the lengths of the pendant paths are $s_{1}, s_{2}, s_{3}, s_{4}$ or $s_{1}, s_{2}, s_{3}, s_{4}, s_{5}$ in case $l_{3}=1$. Figure 4 shows one possibility for $G$, namely, when the middle cycle is $C_{6}\left(P_{1}, P_{s_{1}+1}, P_{s_{2}+1}, P_{1}, P_{s_{3}+1}, P_{s_{4}+1}\right)$, i.e., $l_{3}=4$.

Let $G^{\prime \prime}=\Phi_{6}^{I}\left(l_{1}^{\prime}, l_{2}^{\prime}+s_{1}+s_{2}+s_{3}+s_{4} ; 2\right)$ or $\Phi_{6}^{I}\left(l_{1}^{\prime}, l_{2}^{\prime}+s_{1}+s_{2}+s_{3}+s_{4}+s_{5} ; 2\right)$ when $l_{3}=1$. It suffices to show that if $l_{3} \neq 2$ or $l_{3}=2$ and $s_{1}+s_{2}+s_{3}+s_{4} \geq 1$, then $G \prec G^{\prime \prime}$.

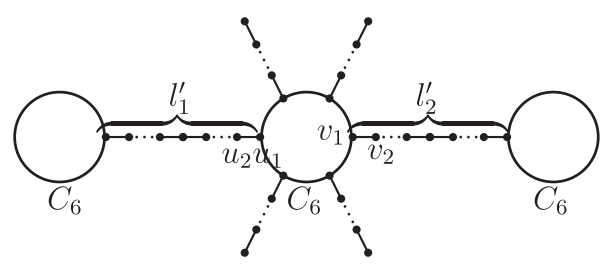

Figure 4: $G\left(l_{3}=4\right)$. 


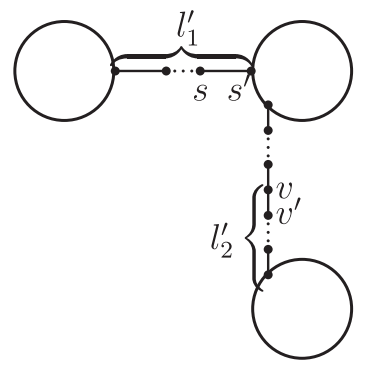

Figure 5: $G^{\prime \prime}$.

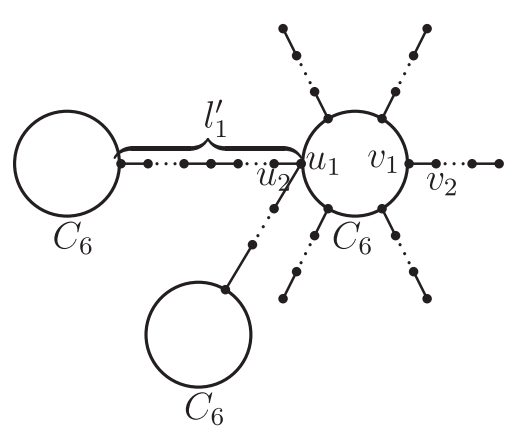

Figure 6: $G\left(l_{3}=1\right)$.

Now take $G_{1}$ to the graph obtained by connecting a $C_{6}$ and the middle sun graph of $G$ by a path $P_{l_{2}^{\prime}}$ and take $G_{2}$ to the graph obtained by connecting two $C_{6}^{\prime} s$ by a path $P_{l_{2}^{\prime}+s_{1}+\cdots+s_{4}}$ (or $P_{l_{2}^{\prime}+s_{1}+\cdots+s_{5}}$ in case $l_{3}=1$ ). By Lemma 2.1 one readily shows that $G_{1} \leq G_{2}$, and with strict inequality if $\sum s_{i} \geq 1$. Refer to Figure 4 or Figure 6 for graph $G$ and to Figure 5 for graph $G^{\prime \prime}$. Now take $H$ to be $E_{6, l_{1}^{\prime}+4}$ and apply Lemma 2.1 with $G, G^{\prime \prime}, u_{1}, s^{\prime}, u_{2}$ (and $s$ ) playing the role of $G, G^{\prime}, u, u^{\prime}, v$ respectively. when $l_{3} \neq 1, G_{1}-u_{1}$ is a unicyclic graph and $G_{2}-s^{\prime}$ is a lollipop graph of the same order. So $G_{1}-u_{1} \preceq G_{2}-s^{\prime}$ and with strict inequality, if $G_{1}-u_{1}$ is not a lollipop, i.e., provided that $l_{3} \neq 2$ or $l_{3}=2$ and $s_{1}+\cdots+s_{4} \geq 1$. As we have noted, $G_{1} \preceq G_{2}$, and with strict inequality when $\sum s_{i} \geq 1$. So by Lemma 2.1 we obtain $G \prec G^{\prime \prime}$. When $l_{3}=1, G_{2}-s^{\prime}$ equals the lollipop graph $E_{6, l_{2}^{\prime}+s_{1}+\cdots+s_{5}+9}$ and $G_{1}-u_{1}$ equals the union of $E_{6, l_{2}^{\prime}+4}$ and a tree, say $T$, of order $s_{1}+\cdots+s_{5}+5$. By adding a suitable edge joining a vertex of $E_{6, l_{2}^{\prime}+4}$ to a vertex of $T$ one obtains a unicyclic graph $F$ with the same order as $G_{2}-s^{\prime}$. Hence $G_{2}-s^{\prime}>G_{1}-u_{1}$. Then by applying Lemma 2.1 we also obtain $G \prec G^{\prime \prime}$ for this case.

Lemma 2.3. For any positive integers $a, b \geq 6$, if $\min \{a, b\}>6$ then $E_{6, a} \cup E_{6, b} \prec C_{6} \cup E_{6, a+b-6}$.

Proof. Without loss of generality, assume that $\min \{a, b\}>6$. By Lemma 1.2 we have

$$
m_{k}\left(E_{6, a} \cup E_{6, b}\right)=m_{k}\left(E_{6, b} \cup C_{6} \cup P_{a-6}\right)+m_{k-1}\left(E_{6, b} \cup P_{a-7} \cup P_{5}\right)
$$




$$
m_{k}\left(C_{6} \cup E_{6, a+b-6}\right)=m_{k}\left(E_{6, b} \cup C_{6} \cup P_{a-6}\right)+m_{k-1}\left(E_{6, b-1} \cup P_{a-7} \cup C_{6}\right) .
$$

Now we only need to show that $E_{6, b} \cup P_{5} \prec E_{6, b-1} \cup C_{6}$. We proceed by induction on $b$. When $b=7$, by Lemma 1.2 , we have

$$
\begin{aligned}
m_{k}\left(E_{6,7} \cup P_{5}\right) & =m_{k}\left(C_{6} \cup P_{1} \cup P_{5}\right)+m_{k-1}\left(P_{5} \cup P_{5}\right) \\
& =m_{k}\left(C_{6} \cup P_{1} \cup P_{5}\right)+m_{k-1}\left(P_{5} \cup P_{1} \cup P_{4}\right)+m_{k-2}\left(P_{5} \cup P_{3}\right)
\end{aligned}
$$

and

$$
\begin{aligned}
m_{k}\left(C_{6} \cup C_{6}\right) & =m_{k}\left(C_{6} \cup P_{6}\right)+m_{k-1}\left(C_{6} \cup P_{4}\right) \\
& =m_{k}\left(C_{6} \cup P_{6}\right)+m_{k-1}\left(P_{6} \cup P_{4}\right)+m_{k-2}\left(P_{4} \cup P_{4}\right) .
\end{aligned}
$$

By Lemma 1.5 we have $P_{1} \cup P_{5} \prec P_{6}, P_{5} \cup P_{3} \prec P_{4} \cup P_{4}$. So $E_{6,7} \cup P_{5} \prec C_{6} \cup C_{6}$. For the case when $b=8$, by calculation, we have Table 1 . So we have $E_{6,8} \cup P_{5}<E_{6,7} \cup C_{6}$.

Table 1: The $k$-matching numbers of $E_{6,8} \cup P_{5}$ and $E_{6,7} \cup C_{6}$ for $k=1, \ldots, 6$.

\begin{tabular}{lcccccc}
\hline$k=$ & 1 & 2 & 3 & 4 & 5 & 6 \\
\hline$m_{k}\left(E_{6,8} \cup P_{5}\right)$ & 12 & 54 & 114 & 115 & 50 & 6 \\
$m_{k}\left(E_{6,7} \cup C_{6}\right)$ & 13 & 64 & 148 & 161 & 71 & 10 \\
\hline
\end{tabular}

For $b \geq 9$, we have

$$
\begin{aligned}
m_{k}\left(E_{6, b} \cup P_{5}\right) & =m_{k}\left(E_{6, b-1} \cup P_{5}\right)+m_{k-1}\left(E_{6, b-2} \cup P_{5}\right) \\
m_{k}\left(E_{6, b-1} \cup C_{6}\right) & =m_{k}\left(E_{6, b-2} \cup C_{6}\right)+m_{k-1}\left(E_{6, b-3} \cup C_{6}\right) .
\end{aligned}
$$

By induction, we have $E_{6, b-1} \cup P_{5} \prec E_{6, b-2} \cup C_{6}$ and $E_{6, b-2} \cup P_{5} \prec E_{6, b-3} \cup C_{6}$; hence $E_{6, b} \cup P_{5} \prec$ $E_{6, b-1} \cup C_{6}$.

Lemma 2.4. For any positive integers $l_{1}, l_{2} \geq 2$ with $l_{1}+l_{2}=n-14$, if $\min \left\{l_{1}, l_{2}\right\}>2$ then $\Phi_{6}^{I}\left(l_{1}, l_{2} ; 2\right) \prec \Phi_{6}^{I}(n-16,2 ; 2)$.

Proof. For brevity, we denote $\Phi_{6}^{I}\left(l_{1}, l_{2} ; 2\right)$ and $\Phi_{6}^{I}(n-16,2 ; 2)$ respectively by $G$ and $H$. Referring to Figure 7 , by Lemma 1.2 we have

$$
\begin{aligned}
& m_{k}(G)=m_{k}\left(G-u_{1} v_{1}\right)+m_{k-1}\left(E_{6, l_{1}+4} \cup E_{6, l_{2}+4} \cup P_{4}\right), \\
& m_{k}(H)=m_{k}\left(H-u_{0} v_{0}\right)+m_{k-1}\left(E_{6, n-12} \cup C_{6} \cup P_{4}\right) .
\end{aligned}
$$

Because $G-u_{1} v_{1}=H-u_{0} \nu_{0}$ and $E_{6, l_{1}+4} \cup E_{6, l_{2}+4} \prec E_{6, n-12} \cup C_{6}$ by Lemma 2.3, we obtain $G \prec H$.

Lemma 2.5. For any graph $G \in G_{6, n}^{\Pi} \backslash \Phi_{6}^{\Pi}(n-17,2,2)$ with $n \geq 19, G<\Phi_{6}^{\Pi}(n-17,2,2)$. 

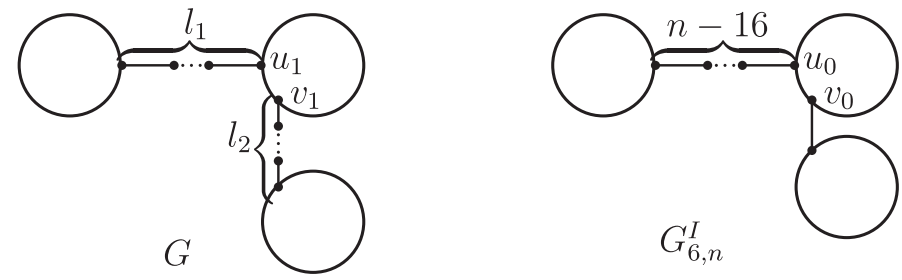

Figure 7: The graphs $G$ and $H$.

Proof. First, using Lemma 2.1, we can show that for any graph $G \in G_{6, n}^{\Pi} \backslash \Phi_{6, n}^{\Pi}, G \prec \Phi_{6}^{\Pi}\left(l_{1}, l_{2}, l_{3}\right)$ for some positive integers $l_{1}, l_{2}, l_{3}$ with $\min \left\{l_{1}, l_{2}, l_{3}\right\} \geq 2$. Thus hereafter we assume that $G=$ $\Phi_{6}^{\Pi}\left(l_{1}, l_{2}, l_{3}\right)$. If $\min \left\{l_{1}, l_{2}, l_{3}\right\}>2$, then we show that $\Phi_{6}^{\Pi}\left(l_{1}, l_{2}, l_{3}\right) \prec \Phi_{6}^{\Pi}\left(l_{1}^{\prime}, l_{2}^{\prime}, 2\right)$ for some positive integers $l_{1}^{\prime}, l_{2}^{\prime} \geq 2$ with $l_{1}^{\prime}+l_{2}^{\prime}=n-15$ as follows: Let $G$ and $H=\Phi_{6}^{\Pi}\left(l_{1}, l_{2}^{\prime}, 2\right)$ with $l_{1}+l_{2}^{\prime}=$ $n-15$ be as given in Figure 8. Without loss of generality, assume that $l_{3}=\min \left\{l_{1}, l_{2}, l_{3}\right\}>2$. By
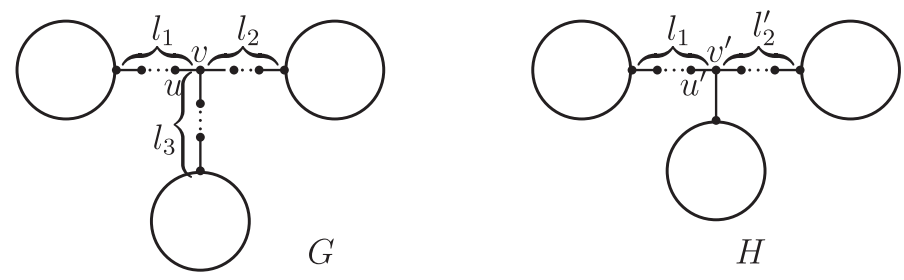

Figure 8: The graphs $G$ and $H$.

Lemma 1.2 we have

$$
\begin{aligned}
& m_{k}(G)=m_{k}(G-u v)+m_{k-1}\left(E_{6, l_{1}+3} \cup E_{6, l_{2}+4} \cup E_{6, l_{3}+4}\right) . \\
& m_{k}(H)=m_{k}\left(H-u^{\prime} v^{\prime}\right)+m_{k-1}\left(E_{6, l_{1}+3} \cup E_{6, l_{2}^{\prime}+4} \cup C_{6}\right) .
\end{aligned}
$$

Note that $G-u v=H-u^{\prime} v^{\prime}$ and by Lemma $2.3, E_{6, l_{2}+4} \cup E_{6, l_{3}+4} \prec E_{6, l_{2}^{\prime}+4} \cup C_{6}$. Hence $\Phi_{6}^{\Pi}\left(l_{1}, l_{2}, l_{3}\right)$ $\prec \Phi_{6}^{\Pi}\left(l_{1}, l_{2}^{\prime}, 2\right)$ and we may assume that $G=\Phi_{6}^{\Pi}\left(l_{1}, l_{2}, 2\right)$ for some positive integers $l_{1}, l_{2} \geq 2$ with $l_{1}+l_{2}=n-15$.

If $\min \left\{l_{1}, l_{2}\right\}>2$, then we prove that $\Phi_{6}^{\Pi}\left(l_{1}, l_{2}, 2\right) \prec \Phi_{6}^{\Pi}(n-17,2,2)$. Let $G$ and $H=\Phi_{6}^{\Pi}(n-$ $17,2,2)$ be as shown in Figure 9.

By Lemma 1.2 we have

$$
\begin{aligned}
& m_{k}(G)=m_{k}(G-u v)+m_{k-1}\left(E_{6, l_{1}+4} \cup E_{6, l_{2}+4} \cup P_{5}\right), \\
& m_{k}(H)=m_{k}\left(H-u^{\prime} v^{\prime}\right)+m_{k-1}\left(E_{6, l_{1}+l_{2}+2} \cup C_{6} \cup P_{5}\right) .
\end{aligned}
$$

Since we have $G-u v=H-u^{\prime} v^{\prime}$ and $E_{6, l_{1}+4} \cup E_{6, l_{2}+4} \prec E_{6, l_{1}+l_{2}+2} \cup C_{6}$ by Lemma 2.3, we are done. 


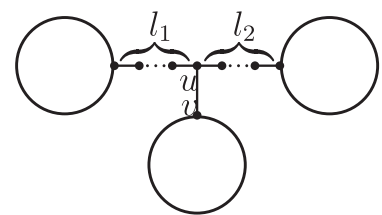

G

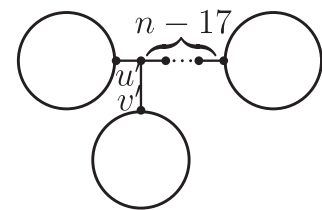

$H$

Figure 9: The graphs $G$ and $H$.

Lemma 2.6. For $n \geq 19$, we have $M E\left(\Phi_{6}^{I}(n-16,2 ; 2)\right)<M E\left(\Phi_{6}^{I I}(n-17,2,2)\right)$ and, moreover, $\Phi_{6}^{I}(n-16,2 ; 2) \prec \Phi_{6}^{I I}(n-17,2,2)$ except when $n=20,22$.

Proof.For convenience, let $G=\Phi_{6}^{I}(n-16,2 ; 2)$ and $H=\Phi_{6}^{I I}(n-17,2,2)$, as shown in Figure 10.

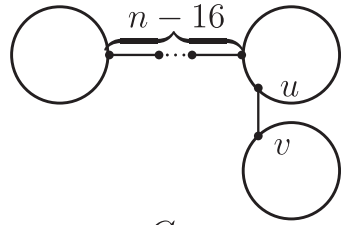

$G$

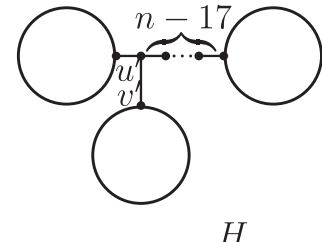

$H$

Figure 10: The graphs $G$ and $H$.

First, assume that $n \geq 20$. By Lemma 1.2 we have

$$
\begin{aligned}
m_{k}(G) & =m_{k}(G-u v)+m_{k-1}(G-u-v) \\
& =m_{k}(G-u v)+m_{k-1}\left(E_{6, n-7} \cup P_{5}\right) \\
& =m_{k}(G-u v)+m_{k-1}\left(E_{6, n-13} \cup P_{6} \cup P_{5}\right)+m_{k-2}\left(E_{6, n-14} \cup P_{5} \cup P_{5}\right), \\
m_{k}(H) & =m_{k}\left(H-u^{\prime} v^{\prime}\right)+m_{k-1}\left(H-u^{\prime}-v^{\prime}\right) \\
& =m_{k}\left(H-u^{\prime} v^{\prime}\right)+m_{k-1}\left(E_{6, n-13} \cup C_{6} \cup P_{5}\right) \\
& =m_{k}\left(H-u^{\prime} v^{\prime}\right)+m_{k-1}\left(E_{6, n-13} \cup P_{6} \cup P_{5}\right)+m_{k-2}\left(E_{6, n-13} \cup P_{4} \cup P_{5}\right) .
\end{aligned}
$$

Since $G-u v=H-u^{\prime} v^{\prime}$, we only need to consider $E_{6, n-14} \cup P_{5} \prec E_{6, n-13} \cup P_{4}$. By Lemma 1.2 we have

$$
\begin{aligned}
& m_{k}\left(E_{6, n-14} \cup P_{5}\right)=m_{k}\left(P_{n-14} \cup P_{5}\right)+m_{k-1}\left(P_{n-20} \cup P_{4} \cup P_{5}\right) \\
& m_{k}\left(E_{6, n-13} \cup P_{4}\right)=m_{k}\left(P_{n-13} \cup P_{4}\right)+m_{k-1}\left(P_{n-19} \cup P_{4} \cup P_{4}\right) .
\end{aligned}
$$

Note that the assumption $n \geq 20$ guarantees that $E_{6, n-14}$ is defined. By Lemma 1.5 (iii) we have $P_{n-14} \cup P_{5} \prec P_{n-13} \cup P_{4}$, provided that $5 \leq n-14$ and $4 \leq n-13$, i.e., $n \geq 19$. Since we are assuming $n \geq 20$, the said quasi-inequality holds. Similarly, we have $P_{n-20} \cup P_{5} \prec P_{n-19} \cup P_{4}$, 
provided that $n \geq 25$. If $n=24, P_{n-20} \cup P_{5}$ and $P_{n-19} \cup P_{4}$ are both equal to $P_{4} \cup P_{5}$. If $n=$ 23, we have $P_{n-20} \cup P_{5}=P_{3} \cup P_{5} \prec P_{n-19} \cup P_{4}=P_{4} \cup P_{4}$ by Lemma 1.5 (iii). If $n=22$, then $P_{n-20} \cup P_{5}>P_{3} \cup P_{4}=P_{n-19} \cup P_{4}$. Similarly, we have $P_{n-20} \cup P_{5} \prec P_{n-19} \cup P_{4}$ for $n=21$ and $P_{n-20} \cup P_{5} \prec P_{n-19} \cup P_{4}$ for $n=20$. So for $n \geq 20$, we have $P_{n-20} \cup P_{5} \leq P_{n-19} \cup P_{4}$ and hence $E_{6, n-14} \cup P_{5} \prec E_{6, n-13} \cup P_{4}$ except for $n=20,22$. Thus for $n \geq 20$, we always have $G<H$ except possibly when $n=20,22$.

Now we treat the case $n=19$. We have

$$
\begin{aligned}
m_{k}(G) & =m_{k}(G-u v)+m_{k-1}(G-u-v) \\
& =m_{k}(G-u v)+m_{k-1}\left(E_{6,12} \cup P_{5}\right), \\
m_{k}(H) & =m_{k}\left(H-u^{\prime} v^{\prime}\right)+m_{k-1}\left(H-u^{\prime}-v^{\prime}\right) \\
& =m_{k}\left(H-u^{\prime} v^{\prime}\right)+m_{k-1}\left(C_{6} \cup C_{6} \cup P_{5}\right) .
\end{aligned}
$$

Note that $G-u v=H-u^{\prime} v^{\prime}$, so we only need to establish $E_{6,12} \prec C_{6} \cup C_{6}$. Now for all positive integers $k$, since

$$
\begin{aligned}
m_{k}\left(E_{6,12}\right) & =m_{k}\left(P_{12}\right)+m_{k-1}\left(P_{4} \cup P_{6}\right) \\
& =m_{k}\left(P_{6} \cup P_{6}\right)+m_{k-1}\left(P_{5} \cup P_{5}\right)+m_{k-1}\left(P_{4} \cup P_{6}\right) \\
& =m_{k}\left(P_{6} \cup P_{6}\right)+m_{k-1}\left(P_{4} \cup P_{5}\right)+m_{k-2}\left(P_{3} \cup P_{5}\right)+m_{k-1}\left(P_{4} \cup P_{6}\right) \\
& \leq m_{k}\left(P_{6} \cup P_{6}\right)+m_{k-1}\left(P_{4} \cup P_{6}\right)+m_{k-2}\left(P_{4} \cup P_{4}\right)+m_{k-1}\left(P_{4} \cup P_{6}\right) \\
& =m_{k}\left(C_{6} \cup C_{6}\right),
\end{aligned}
$$

where at least one inequality holds strictly, we are done. So we also obtain $G \prec H$ in this case.

For $n=20$, and $n=22$, by calculation we obtain Tables 2 and 3. So, for these cases, we have neither $G \preceq H$ nor $G \succeq H$. Making use of Theorem 1.1, we also obtain $\operatorname{ME}(G)=30.0168$ and $M E(H)=30.0334$ when $n=20$, and $M E(G)=31.877$ and $M E(H)=31.9742$ when $n=22$. In either case, we have $M E(G)<M E(H)$.

Table 2: The $k$-matching numbers of $G$ and $H$ for $k=1, \ldots, 10$.

\begin{tabular}{lcccccccccc}
\hline$k=$ & 1 & 2 & 3 & 4 & 5 & 6 & 7 & 8 & 9 & 10 \\
\hline$m_{k}(G)$ & 22 & 203 & 1014 & 4239 & 5683 & 6311 & 4008 & 1321 & 191 & 8 \\
$m_{k}(H)$ & 22 & 203 & 1024 & 4238 & 5674 & 6281 & 3964 & 1296 & 188 & 8 \\
\hline
\end{tabular}

The proof is completed.

In conclusion, we obtain the following result:

Theorem 2.7. For any graph $G \in G_{6, n} \backslash\left\{\Phi_{6}^{I I}(n-17,2,2)\right\}$ with $n \geq 19$, we have $M E(G)<M E$ $\left(\Phi_{6}^{I I}(n-17,2,2)\right)$ and, moreover, $G \prec \Phi_{6}^{I I}(n-17,2,2)$ except when $n=20,22$ and $G=\Phi_{6}^{I}(n-$ $16,2 ; 2)$. 
Table 3: The $k$-matching numbers of $G$ and $H$ for $k=1, \ldots, 11$.

\begin{tabular}{lccccccccccc}
\hline$k=$ & 1 & 2 & 3 & 4 & 5 & 6 & 7 & 8 & 9 & 10 & 11 \\
\hline$m_{k}(G)$ & 24 & 246 & 1410 & 4760 & 9888 & 14011 & 13600 & 7217 & 1956 & 237 & 8 \\
$m_{k}(H)$ & 24 & 246 & 1410 & 4760 & 9888 & 13594 & 13600 & 7207 & 1964 & 240 & 8 \\
\hline
\end{tabular}

We would like to add that when $n=18, G_{6, n}^{\Pi}$ is empty, $G_{6, n}^{I}=\Phi_{6, n}^{I}$ and $\Phi_{6, n}^{I}(2,2 ; 2)>G$ for every $G \in G_{6, n} \backslash \Phi_{6, n}^{I}(2,2 ; 2)$. When $n<18, G_{6, n}$ is empty.

\section{References}

[1] L. Chen and Y. Shi, The maximal matching energy of tricyclic graphs, MATCH Commun. Math. Comput. Chem., 73 (2015), 105-119.

[2] L. Chen, J. Liu and Y. Shi, Matching energy of unicyclic and bicyclic graphs with a given diameter, Complexity, to appear.

[3] I. Gutman, Graphs with greatest number of matchings, Publ. Inst. Math. (Beograd), 27 (1980), 67-76.

[4] I. Gutman, X. Li, Y. Shi and J. Zhang, Hypoenergetic trees, MATCH Commun. Math. Comput. Chem., 60 (2008), 415-426.

[5] I. Gutman nad S. Wagner, The matching energy of a graph, Discr. Appl. Math., 160 (2012), 2177-2187.

[6] B. Huo, X. Li and Y. Shi, Complete solution to a conjecture on the maximal energy of unicyclic graphs, European J. Combin., 32 (2011), 662-673.

[7] B. Huo, X. Li and Y. Shi, Complete solution to a problem on the maximal energy of unicyclic bipartite graphs, Lin. Algebra Appl., 434(2011), 1370-1377.

[8] S. J. Ji, X. L. Li and Y. T. Shi, Extremal matching energy of bicyclic graphs, MATCH Commun. Math. Comput. Chem., 70 (2013), 697-706.

[9] H. H. Li, B. S. Tam and L. Su, On the signless Laplacian coeffcients of unicyclic graphs, Lin. Algebra Appl., 439 (2013), 2008-2009.

[10] H. H. Li, Y. X. Zhou, L. Su, Graphs with extremal matching energies and prescribed parameters, MATCH Commun. Math. Comput. Chem., 72 (2014), 239-248.

[11] S. L. Li and W. G. Yan, The matching energy of graphs with given parameters, Discr. Appl. Math., 162 (2014), 415-420.

[12] X. Li, Y. Shi, I. Gutman, Graph Energy, Springer, New York, 2012.

[13] X. L. Li, Y. T. Shi and M. Q. Wei, On a conjecture about tricyclic graphs with maximal energy, MATCH Commun. Math. Comput. Chem., 72 (2014), 183-214.

[14] K. Xu, K. C. Das and Z. Zheng, The minimal matching energy of $(n, m)$-graphs with a given matching number, MATCH Commun. Math. Comput. Chem., 73 (2015), 93-104.

College of Mathematics and Information Science, Jiangxi Normal University, Nanchang, Jiangxi 330022, China.

E-mail: yunxia2008@163.com

College of Mathematics and Information Science, Jiangxi Normal University, Nanchang, Jiangxi 330022, China.

E-mail: lhh@jxnu.edu.cn 